\title{
Homothetic mapping as means to obtain a wide field of view: the Delft Testbed Interferometer
}

\author{
Hedser van Brug, Bastiaan Oostdijck, Bart Snijders, Cas van der Avoort ${ }^{\dagger}$, Pierre-Marie Gori ${ }^{\ddagger}$
}

TNO TPD, Stieltjesweg 1, 2628 CK Delft, the Netherlands

†SRON - TU Delft, the Netherlands

$\ddagger$ Leiden Observatory, University Leiden, the Netherlands

\begin{abstract}
The Delft Testbed Interferometer (DTI) will be presented. The basics of homothetic mapping will be explained together with the method of fulfilling the requirements as chosen in the DTI setup. The optical layout incorporates a novel tracking concept enabling the use of homothetic mapping in real telescope systems for observations on the sky. The requirements for homothetic mapping and the choices made in the DTI setup will be discussed. Finally the first results and the planned experiments will be presented.
\end{abstract}

Keywords: Multi aperture interferometry, wide field imaging, homothetic mapping

\section{INTRODUCTION}

At TPD (the institute of applied physics) of TNO (a Dutch organization founded as an interface between the scientific research at the Dutch universities and the knowledge demands of the Dutch industries), there is a research effort going on indicated as the Knowledge center for Aperture Synthesis (KAS).

KAS is a joined effort of TNO TPD with Space Research Organization Netherlands (SRON), Observatory Leiden, Delft University of Technology, European Space Agency (ESA), and European Southern Observatory (ESO). Presently running research includes many investigations in the field of optical aperture synthesis.

KAS research involves a large number of topics, e.g. nulling interferometry, adaptive optics, delay line systems (small for Darwin, and large for VLT-I), and the topic for this paper, homothetic mapping. Homothetic mapping is one of the methods that can be used to combine the light from several telescopes optically. The important benefit of homothetic mapping is that it allows for a large field of view. This is beneficial for multi telescope systems like VLT-I in Chile and the ESA Darwin mission, but may in the future also become an important technology for earth observing satellites. Instead of observing single stars only, homothetic mapping opens the possibility to observe multiple stars and extended structures, simultaneously.

The Delft Testbed Interferometer, a setup to demonstrate the feasibility of homothetic mapping, is intended as a test facility to investigate all the aspects of optical aperture synthesis, with emphasis on homothetic mapping. The lessons learned from investigations on DTI will be used during the development of homothetic mappers for both earth based and space based observatories.

This paper deals with the basics behind homothetic mapping, the requirements it poses on the optical layout, the implementation of homothetic mapping in the Delft Testbed Interferometer, and the first results.

Further author information:

Hedser van Brug: E-mail: brug@tpd.tno.nl, Telephone: +31 152692489 


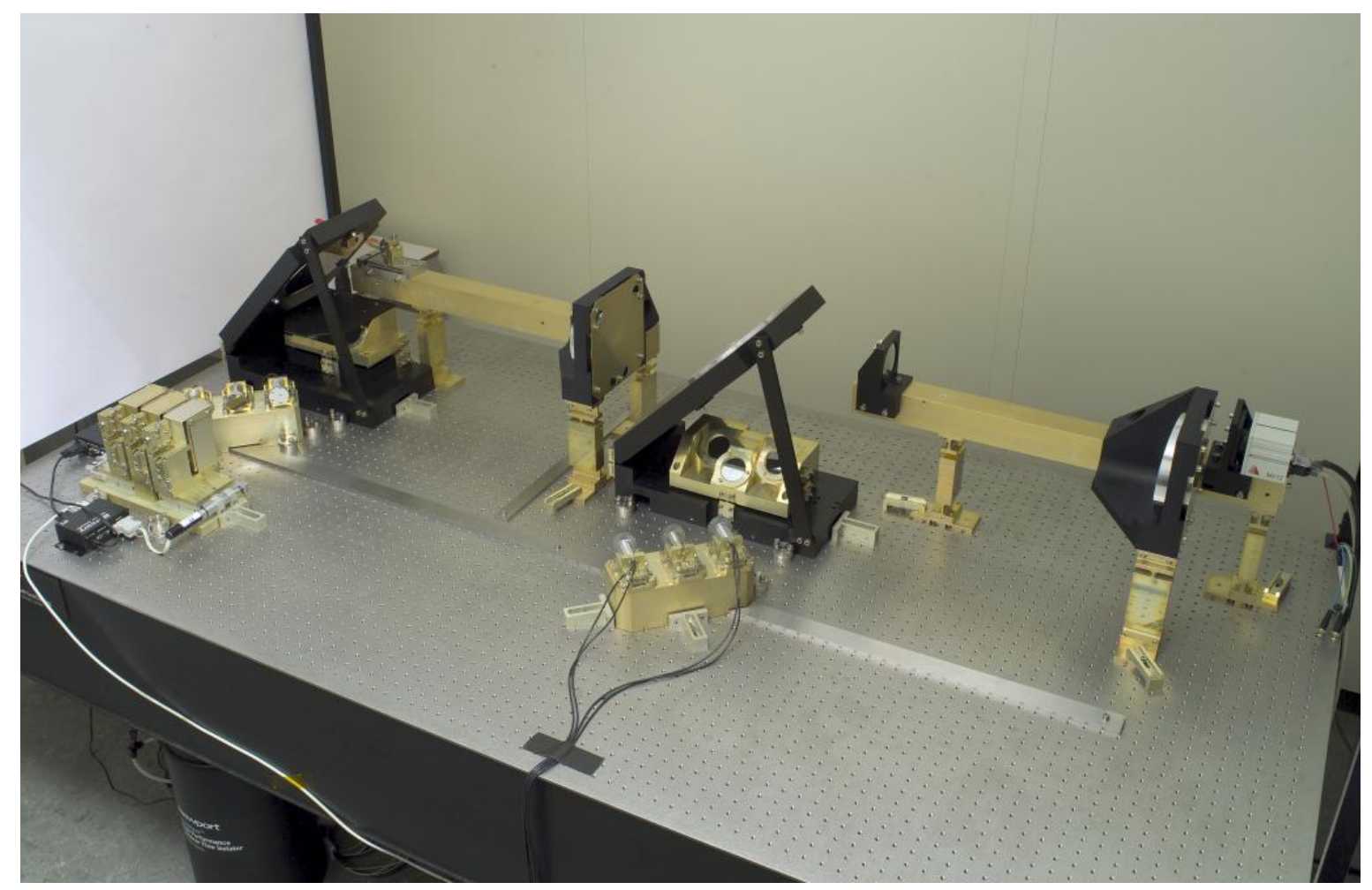

Figure 1. Photograph of the assembled, fully aligned, and operational DTI setup. On the left hand side the star simulator, the aperture plate, the delay lines and the first part of the mapping mirrors can be seen. On the right hand side the remainder of the mapping mirrors is visible, together with the imaging telescope.

\section{HOMOTHETIC MAPPING}

When a multi telescope system is used as an interferometer, the optical outputs of all the telescopes are combined, after appropriate scaling, in an imaging beam combiner. The principle of homothetic mapping is that the arrangement of the beams that are combined and their diameters, are a scaled copy of the arrangement of the telescopes and the size of their primary mirrors. To arrive at a higher resolving power of the combined beams, in comparison to a single beam, the combined beams should interfere. To this end they should experience differences in optical path length that are short as compared to the wavelength of the light used. Exact requirement in terms of path length equality depends on the wavelength range, e.g. when a laser is used several milimeters difference can be allowed (depending on the coherence length) while for a wide wavelength band source more than a few micro meters is already too much.

The benefit of multi aperture interferometry over the use of single telescopes can be made clear by the following consideration. The width of the interference fringes is defined by the baseline of the system, i.e. the separation between the telescopes, and not by the size of the individual telescopes. In case that the baseline is ten times the size of an individual telescope, the fringes will be one tenth of the size of the Airy spot (the image formed by a diffraction limited optical system of a point like star). This leads to a resolution that is ten times better.

In order to investigate the ins and outs of homothetic mapping a breadboard system has been designed and built, the Delft Testbed Interferometer, as shown in Fig.1.

DTI is intended as a research instrument for $\mathrm{PhD}$ students in the field of optical aperture synthesis and is available as a guest facility. 


\section{OPTICAL LAYOUT}

In this section the optical layout will be presented and the choices made will be explained. Since DTI is created for the investigation of optical aperture synthesis concepts, and especially homothetic mapping, it has to consist of a star simulator, the multi aperture telescope system, a mapping arrangement and finally an imaging telescope. Each of these components will be discussed in the following subsections. An overview of the system is shown in Fig.1.

\subsection{Star-simulator}

As light source for the stars we have selected a Xe arc lamp. It emits light over the full visible range. The emitted light is coupled into three single mode fibres, of which the cutoff wavelength equals $460 \mathrm{~nm}$. The exit facets of these fibres are placed in the focal plane of a parabolic mirror. This parabolic mirror is used to collimate the light coming out of the fibres. Each fibre represents an ideal point source, our stars, and the stars are placed virtually at infinite via the parabolic mirror. Since the fibres are placed with a spacing between them in the order of several hundreds of micrometers (up to $600 \mu \mathrm{m}$ ), and the focal length of the parabolic mirror is $600 \mathrm{~mm}$, the angles between the collimated light beams are approximately $1 \mathrm{mrad}(206 \mathrm{arcsec})$. This angle is at the moment the maximum field of view (FoV) of DTI and is mainly limited by the size of the camera, behind the beam combiner/imaging telescope, on which the stars are imaged.

\subsection{Multi aperture telescope system}

The light coming from the parabolic mirror is reflected downwards by a large, flat mirror (see Fig.1). The light passes then through an aluminum aperture plate in which three $1 \mathrm{~cm}$ diameter holes are made, simulating three telescopes. Below these three apertures there are three mirrors that direct the light towards the roof top design delay lines, see Fig.2. These delay lines allow us to compensate for possible pupil rotations, one of the possible errors in a homothetic mapping system. The stroke of the delay lines is $25 \mathrm{~mm}$ with an accuracy of about $1 \mu \mathrm{m}$. The delay line in the middle is not actuated. That beam path is taken to define the reference optical path length. The outer beams should be adjusted such as to obtain an overall path length that is equal to the length of the central beam path.

\subsection{Mapping arrangement}

The main objective in homothetic mapping is to arrive at a beam layout in the beam combiner that is a scaled copy of the telescope layout (in our case the layout of the apertures). Since the goal of the DTI setup is to proof that homothetic mapping is possible we have selected a layout that is easy to align. To this end we have created a mapping stage where three mirror, the mapping mirrors, are aligned equal to the three mirrors beneath the aperture plate. The only requirement now is to let the three beams per star reflect at these mirrors with an arrangement that is equal to how they are defined by the apertures. The beams can be shifted over the mirrors via a periscope arrangement as can be seen in Figs.1 and 3. Each of the three sub beams is guided over two flat mirrors that are mutually parallel. By tip/tilting these two mirrors while they remain parallel, the position at which the beams hit the mapping mirrors can be adjusted, and thus the mapping condition can be fulfilled. Just as for the delay line, the central beam path is taken as reference such that only the two outer mirrors have to be adjusted. Apart from the tip/tilt motion, the piezo actuators can also perform a piston movement, providing the means to control the optical path lengths with an accuracy in the order of nanometers. Finally, these mirrors are also used to obtain an overlap of the three sub beams pertaining to the same star, i.e. they are used to get the right pointing direction.

\subsection{Imaging beam combiner}

The light, emitted from three end facets of single mode fibers, arrives at the imaging beam combiner, where light beams pertaining to the same fiber are mutually parallel. The beam combiner consists of a flat mirror to direct the light again parallel to the optical table, and then a paraboloidal and hyperboloidal mirror used to focus down the beams onto the camera. The angle of incidence of the beams define the exact location on the CCD camera. The effective focal length of the beam combiner equals $5 \mathrm{~m}$. The CCD camera is coupled to a computer on which a MatLab program is running that is used to control the setup and to read in the results. 


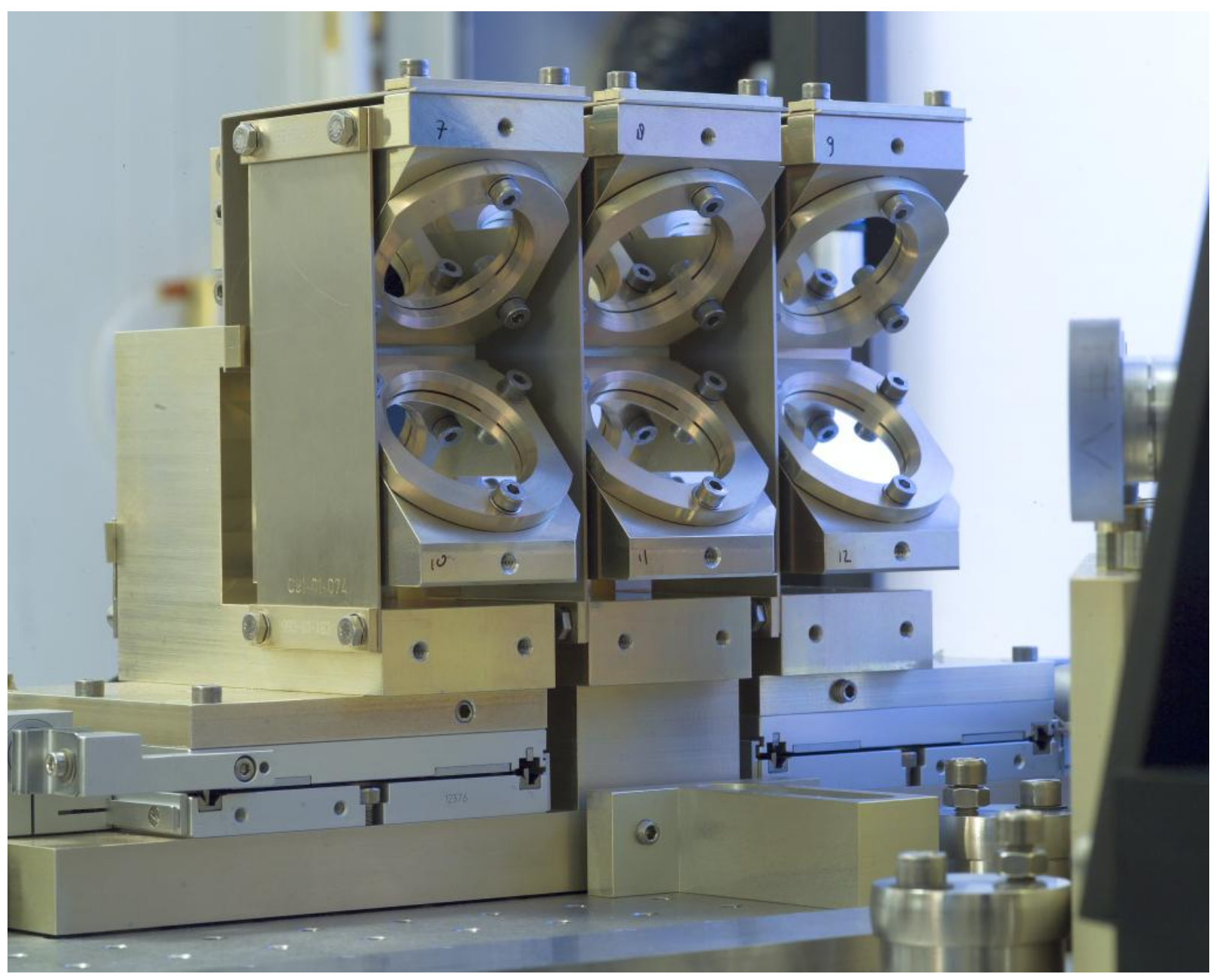

Figure 2. Photograph of the delay lines. These delay lines are aligned with their front faces in one line. Only small adjustments are required to arrive at the zero OPD situation, owing to the chosen optical layout.

\subsection{Layout of components}

Many white light interferometers suffer from the fact that the zero optical path difference (OPD) setting is hard to find. To avoid this problem the layout was designed such that for equal optical path lengths for all three optical paths in the system, all corresponding mirrors for the three paths should be placed in a line. In this way the initial alignment is very straight forward. After mechanically aligning all components it was observed that translations of less than a millimeter of the delay lines are required to arrive at fringes on the star images. By adjusting the piston of the piezo actuated mirrors the fringes can be centered on the star image (for one star). Finally, by adjusting the tip/tilt of the piezo actuated mirrors, and thus by adjusting the mapping, the fringes can be centered on all three stars.

The choice for the two large flat mirrors, placed under 45 degrees to direct the light towards the table, was made in order to be able to demonstrate real sky tracking in the future. By adding degrees of freedom to these large mirrors, and to the three small mirrors beneath each of them, this design enables us to change the viewing direction of the system while remaining in homothetic arrangement. In a real life telescope system the siderostats follow the stars. The viewing directions can in that case be measured. By adjusting the beam directions of the light that is going from the mapping mirrors towards the large flat mirror, the effective baseline 


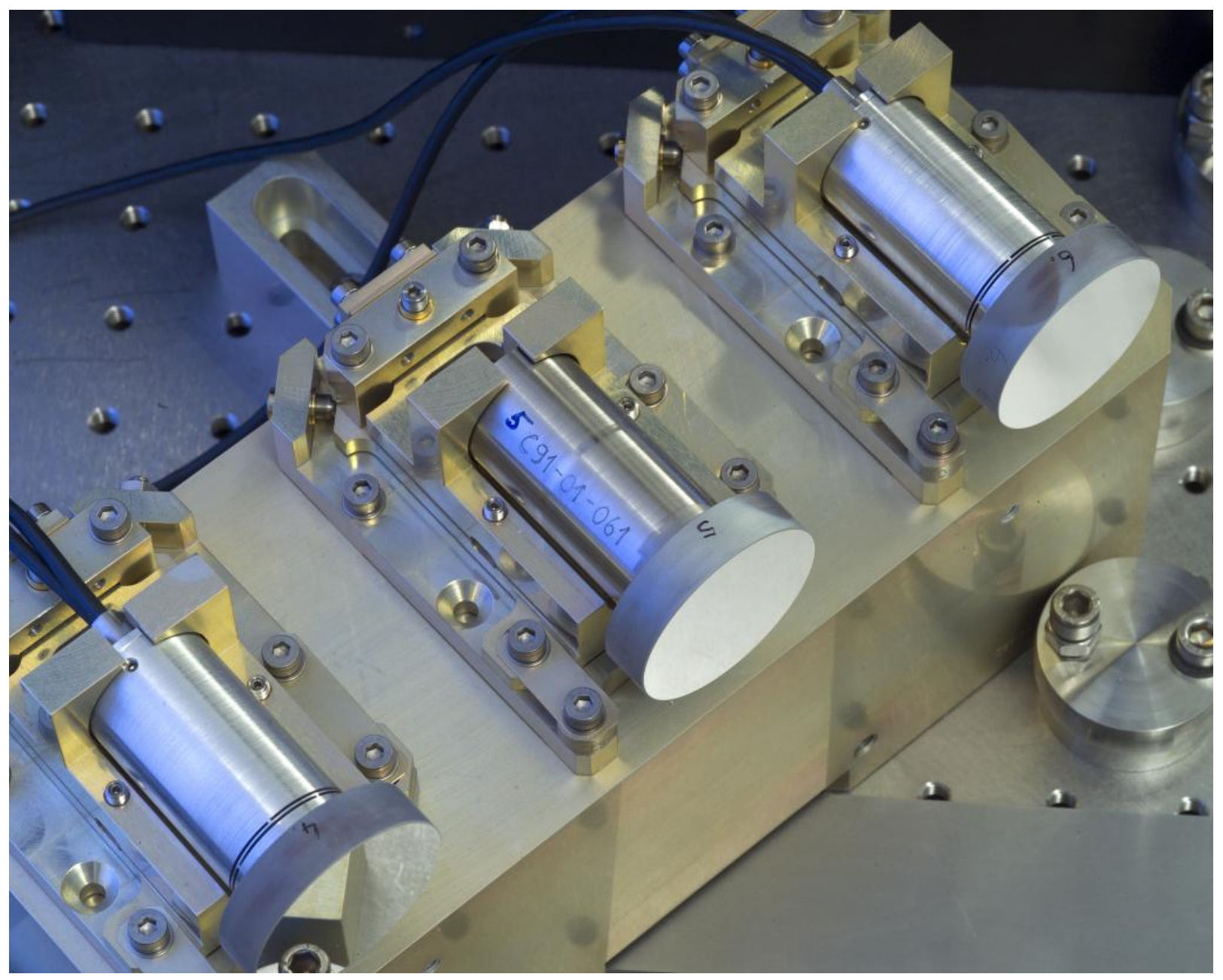

Figure 3. The three mirrors that are used to guide the three beams per star. The central mirror is fixed while the outer mirrors are piezo actuated. They can be tip/tilted and perform a piston movement.

change due to earth rotation is automatically compensated for.

A major benefit from this scheme is that the optical paths within the system will remain stationary during star tracking operation. A graphical impression of this star tracking scheme is depicted in Fig.4. On this automatic mapping during star tracking scheme a patent application is pending.

To compensate for a full 2D star movement the large mirror should have one translational degree of freedom (x-direction in Fig.4) and two rotational degrees of freedom (rotations about the x- and y-directions). The mirrors beneath it, that are aligned in an arrangement that is equal to the telescopes, should also have those three degrees of freedom (translation along the $\mathrm{y}$-direction, and rotations about the $\mathrm{x}$ - and $\mathrm{y}$-directions). The relative arrangement between the mirrors will not have to change. In this way difficult movements of those mirrors, with possible collisions, are not required.

\section{MECHANICAL DESIGN}

The mechanical mountings of the optics have been designed with one goal in mind: the system should be stable. After aligning the system to obtain white light fringes on all three stars the system was found to be stable enough to require only adjustments in the optical path lengths within the micro meter range. The beam 

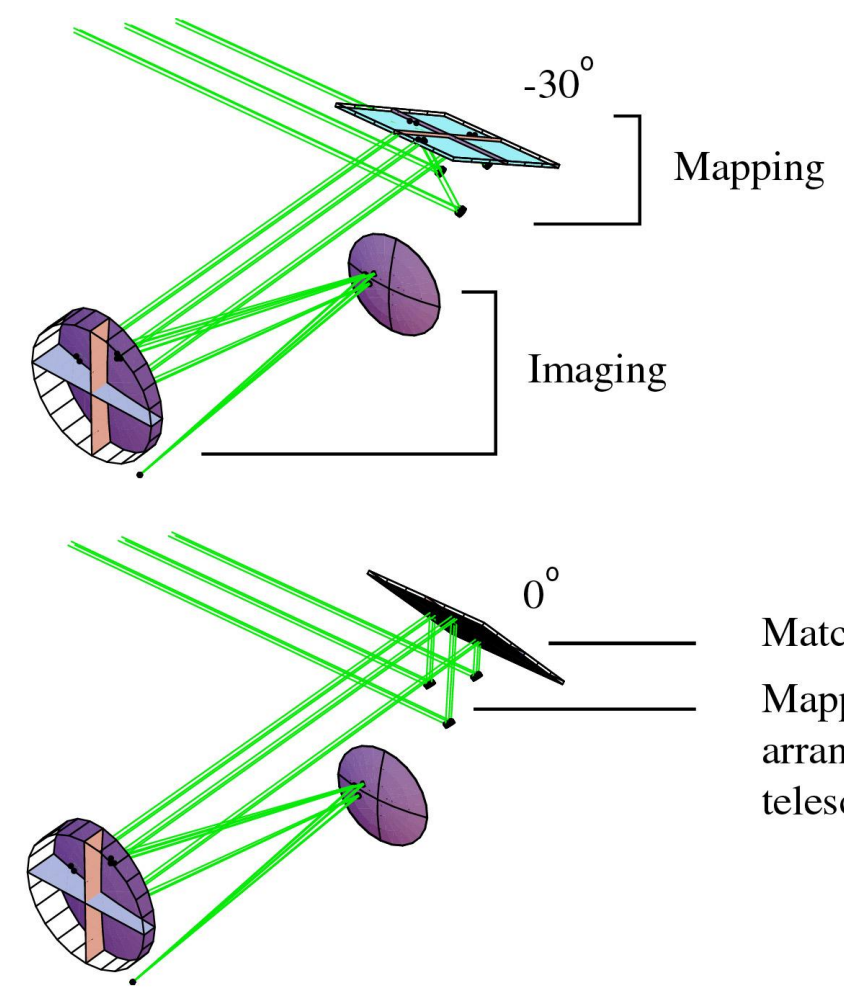

\section{Matching Scan Mirror \\ Mapping Mirrors in scaled arrangement of the telescopes}

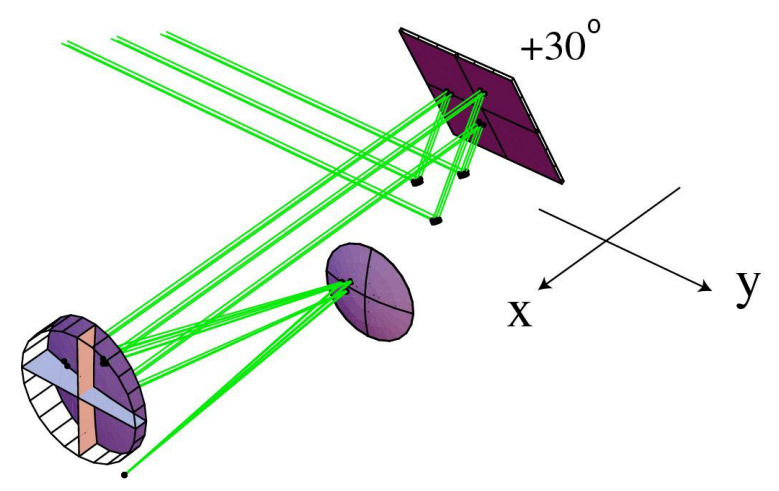

Figure 4. An illustration to show how the large mirror needs to be rotated and scanned to compensate for earth rotations during a long observation. In this figure only the beam combination part is shown. The three light beams originate from three telescopes and have passed the delay lines and the mapping correction mirrors.

pointing was stable enough to have the beams overlap over extended periods of time (days). The requirements for the alignment are: pointing accurate within $3 \mu \mathrm{rad}$, pupil arrangement within $20 \mu \mathrm{m}$, and optical path length equality within $20 \mathrm{~nm}$. The mechanical mountings are stable enough to remain within requirements for the pointing and mapping (pupil arrangement) during several days. The optical path lengths are actively controlled.

\section{FIRST RESULTS}

After constructing all components and mounting all mirrors the alignment could be started. The initial alignment was performed using a HeNe laser as light source. The output of that laser was coupled into a single mode fiber and the end facet of that fiber was placed in the focal plane of the paraboloidal mirror. The beam paths 
were made parallel all the way up to the beam combination telescope. Finally the piezo actuated mirrors are used to obtain a good beam overlap. No more than $10 \%$ misalignment is allowed, according to our error budget as was presented earlier. ${ }^{1}$

After the laser light alignment the beams are parallel throughout the DTI setup, the pointing is therefore correct, and the images per sub beam overlap on the CCD camera.

After finishing the laser light alignment the light of a Xe source was coupled into the single mode fibre. The Xe source supplies us with a broad spectral distribution, it is a white light source. The only goal during this alignment step was to obtain a zero OPD situation. By scanning the coarse delay lines the beam paths could be made equal to within one micrometer. Once adjusted, the coarse delay lines were found to be not needed anymore since the system is very stable. The path lengths could be matched even to the nanometer region by using the piston adjustment possibilities of the piezo actuated mirrors. These adjustments are required on regular basis due to temperature effects and air movements.

Next the second and third star were added. The light of the same Xe source was coupled into two other single mode fibers. The spot size of the Xe source overfills the dimensions of the single mode fibers allowing us to place several fibers in the image of the Xe source.

The images of the three stars should have fringes on them simultaneously, indicating that the system was homothetically mapped. Directly after inserting the light from three stars into the system we observed fringes on all star images. The fringes were not aligned with respect to the envelope of the star image (in our case an Airy pattern). An impression of how the three star images are captured on the CCD camera is shown in Fig.5.

From the fringe positions with respect to the envelope of the Airy disk (the image of the stars in case of a point source), we can calculate the required adjustments to the setup. The OPD errors and the mapping errors can be calculated, enabling a mode of operation where only the fringes on the star images are observed and based on those measurements all modifications are made. A screen capture of the MatLab program that is used to control the system is shown in Fig.6. The length of the pointers in the bottom right hand picture in this figure indicates the amount of off-centricity, while the angle of the pointer gives the direction in which the fringes have shifted. The off-centricity is this figure is far worse than can be obtained by applying the automatic mapping procedure. The shown result has specifically been selected since it shows very clearly what can be observed while a homothetic mapping system is not yet properly aligned. The OPD/mapping errors are here all within $1 \mu \mathrm{m}$, while the system can be aligned to OPD/mapping errors of well within $50 \mathrm{~nm}$.

It is planned to extend DTI in the next few months with a adjustable baseline system. Both the mirrors beneath the aperture plate and the mapping mirrors can then be translated. This allows for baseline change experiments. The plan is to change the baseline at the entrance and to follow this change with the mapping mirrors based on the observation of the fringes.

The required change is that the mirror blocks that are presently used, where all three mirrors are mounted in one structure, will be replaced by three mirror mountings. Each mirror will have its own tip/tilt arrangement to ensure proper beam parallelism. The mirror mountings can be shifted manually, while the third can be shifted manually and computer driven. The computer driven travel is about $10 \mathrm{~mm}$. In order to allow the mirrors to be shifted, the aperture will be divided into three aperture plates, each mounted right above one of the mirrors at the entrance side of DTI.

The experiments will be conducted in the following way. The computer driven optical mount at the entrance, will be shifted. The corresponding mirror at the mapping side will be adjusted in order to remain in mapping configuration. The required change will not be taken from the applied change at the entrance but will be computed from the fringe shifts on the star images.

A totally different activity for the near future is the investigation on how to implement DTI on a real earth based telescope system. Contact with ESO (The European Southern Observatory) will be made to discuss the possibility for DTI on e.g. VLTI (in Chile). Other earth based multi telescope systems are also possible to be included in the investigations. 


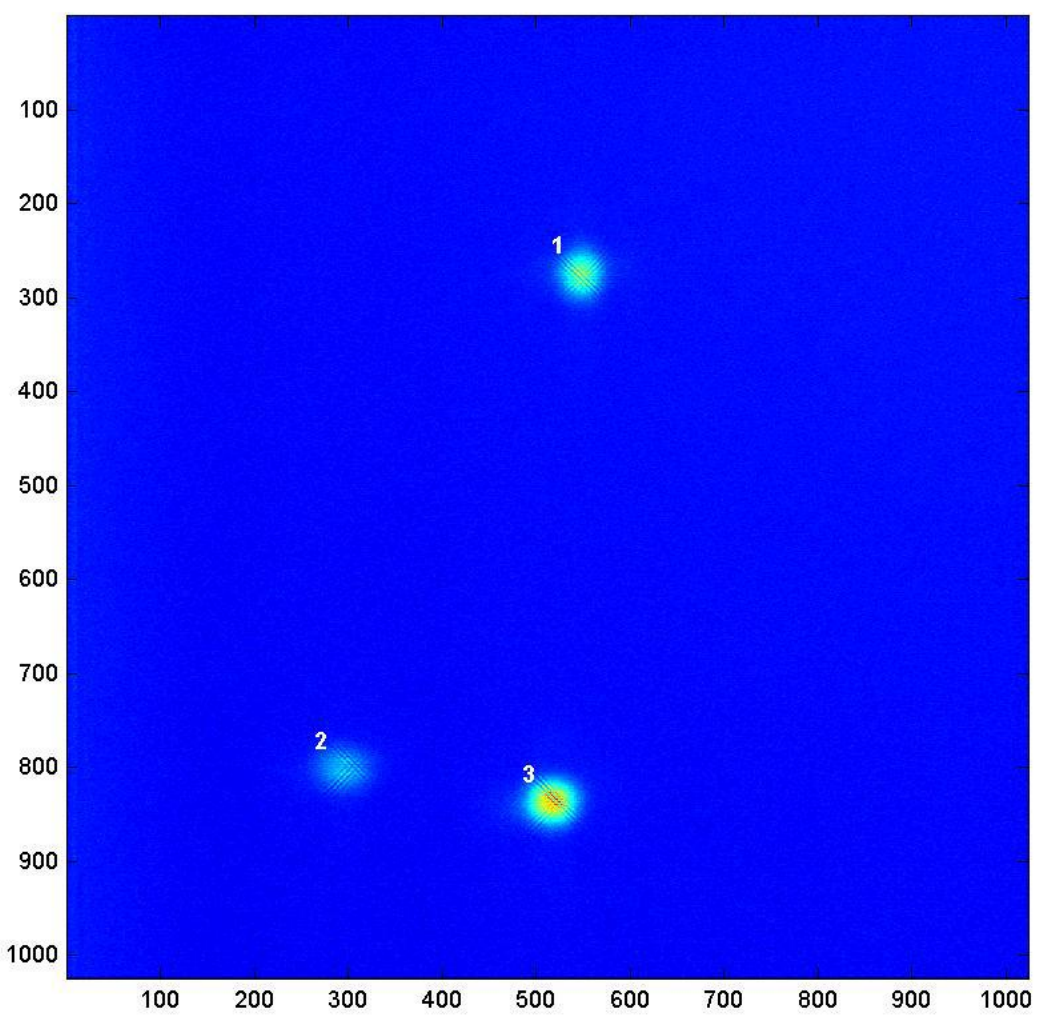

Figure 5. The images of the three stars as recorded by the CCD camera.

\section{CONCLUSIONS}

The Delft Testbed Interferometer has been designed and constructed. After alignment it has been used to demonstrate the feasibility of homothetic mapping. A computer program has been written within the MatLab environment to make the OPD and mapping adjustments automatically. The designed mapping strategy has been shown to be effective. The special arrangement, required to implement homothetic mapping on earth based telescope system has been presented. Using this method no difficult movements are required and the beam paths within the system remain stationary resulting in easier optical path length control. Finally the outlook for DTI is given. An extension is presented allowing for baseline change investigation, and the plans to implement DTI on earth based telescope systems is mentioned.

\section{ACKNOWLEDGMENTS}

The authors want the thank the other members of the Delft Testbed Interferometer team: Wim Gielesen, Teun van den Dool and Peter Giessen. Also Luigi d'Arcio, from ESA and formerly from SRON, needs to be thanked for many useful discussions on the topic of homothetic mapping in general and pupil rotations in specific. 

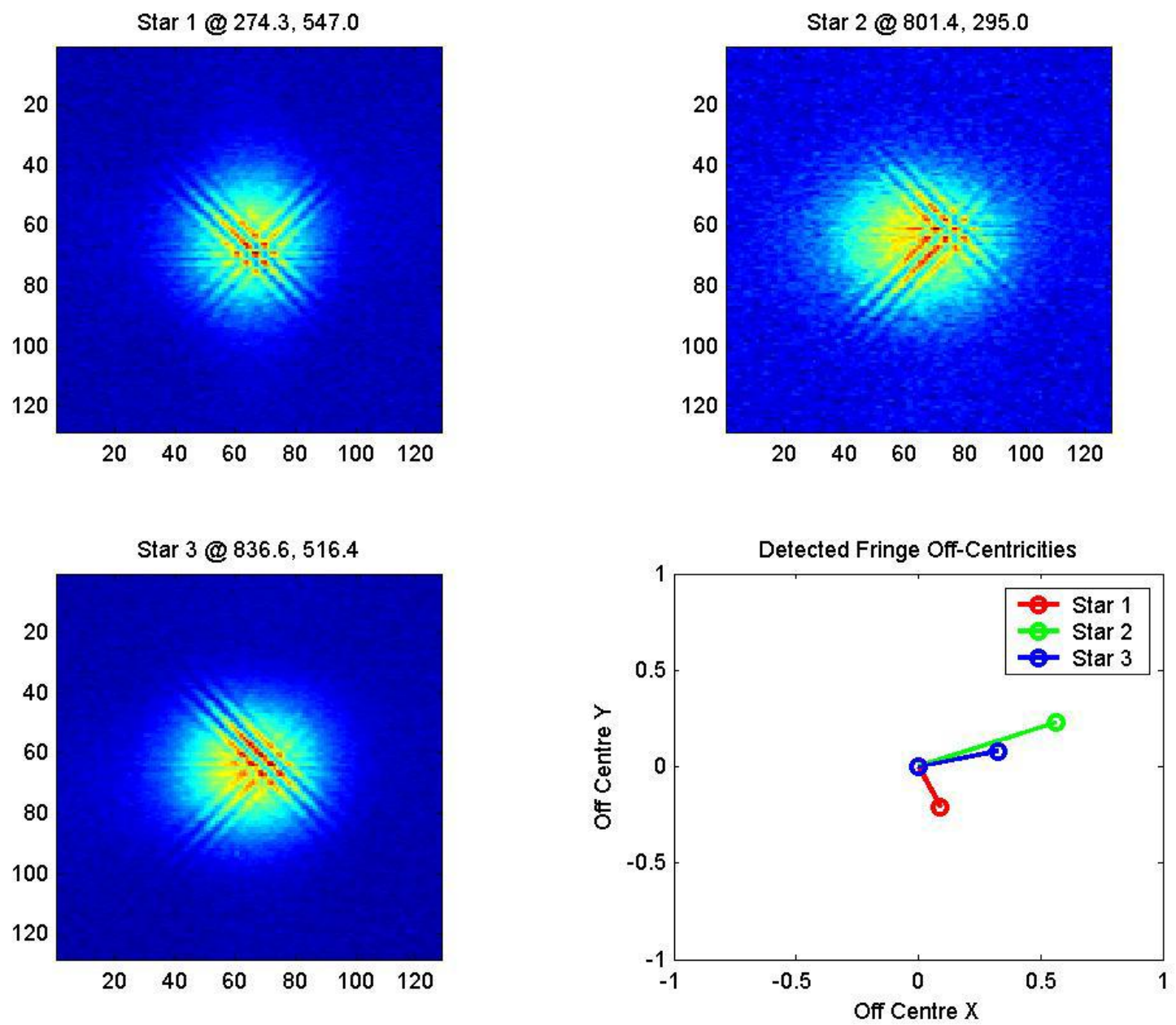

Figure 6. Three sub images selected from Fig.5, together with a graph showing the error in centering the fringes on the envelope of the stars. The off-centricity is a measure for the OPD and mapping errors.

\section{REFERENCES}

1. Hedser van Brug, Teun van den Dool, Wim Gielesen, Peter Giesen, Bastiaan Oostdijck, and Luigi d'Arcio, "Delft Testbed Interferometer - layout design and research goals -", In Interferometry for Optical Astronomy II, edited by Wesley A. Traub, Proceedings of SPIE Vol 4838, pp.425 - 429 